and hand presentation and became impacted. The patient was in labor from 8 to 11 P.M., and finally the labor was so difficult that we had to deliver the child. She passed through labor without a single nonvulsion or even a symptom of one. I left the patient in a rather critical condition three or four days after confinement. I present the case simply to show that the urine was heavily loaded with albumin and yet no convulsions occurred. This case is not the only one that I recall.

Dr. FIsH, Milwaukee, said it was his custom, as soon as his services were engaged, to make an examination of the urine of the pregnant woman in order to determine the presence or absence of albumin. Cases were cited to show that the presence of albumin was not always a safe criterion alone for bringing on premature labor. In the treatment he never uses pilocarpin, as recommended, for the reason that it is liable to produce edema of the lungs, and on this account he considers it a dangerous remedy. In one case of puerperal eclampsia, the woman had a pulse of 140 , but under the continued use of veratrum viride and morphia it dropped to 70 . He believes that if the pulse is reduced to that point and kept there convulsions will not return.

Dr. Gillespre, Tennessee-I use a little chloroform until I get the woman well under the influence of veratrum. I have used Norwood's tincture, commencing with 15 minims hypodermatically, which is repeated in three to five minim doses, and sometimes it requires four or six doses, but very often the second dose is sufficient to stop the convulsions. After the patient is under the influence of the veratrum $I$ stop the administration of chloroform.

As to prophylaxis, I think most cases can be prevented by keeping the bowels well open, attending to the diet, prescribing the proper amount of exercise, and giving the patient something to act on the kidneys. If there is one remedy that I use more than any other it is the hyposulphite of soda in dram doses every three or four hours until it acts on the bowels. When it does not act freely I give a dose of salts. It not only acts on the bowels, but kidneys, and prevents auto-intoxication.

Dr. B. M. Hypes, St. Louis, Mo.--In my opinion, the weak point of our practice as general physicians is that we are constantly allowing our patients to go into the hands of surgeons and consultants by not properly caring for them during pregnancy, and I hope that all physicians will take their patients in their own hands the moment they are pregnant, watch them, and not permit a month or even two weeks to go by, after they are six months advanced in pregnancy, without making a thorough examination of the urine in order to ascertain whether the kidneys are acting normally or not. The urine of the pregnant woman should be frequently examined and the quantity passed in twenty-four hours measured. The solid constituents of the urine should be measured also, in order to determine whether the urates are properly eliminated, and when you use proper remedies and apply them in time you prevent puerperal eclampsia.

Physicians do not always make a proper distinction between the charaater of different forms of puerperal eclampsia. We have hystero-puerperal eclampsia, and epileptiform eclampsia. Furthermore, we have eclampsia during pregnancy from cerebral disease, but it is my belief that 99 cases out of 100 of eclampsia during pregnancy and the puerperal state arise from insufficient action of the kidneys in eliminating the poisons or the toxins in the blood of pregnant women. The different kinds of convulsions must be separated in order to be properly treated. No one would think of giving veratrum viride in a case of hysterical convulsion; neither would the author of the paper attempt to induce premature labor without properly separating and classifying his cases. Much will depend upon the severity of the symptoms or convulsions. Premature labor is produced with the idea of saving the mother first, or both the mother and child if possible.
Dr. Thomason-I desire to thank the members for the free discussion of my paper. I think I have been partially misunderstood. I do not wish the members of the Section to entertain the idea for a moment that because albuminuria is pres. ent in the case of a pregnant woman it is a sufficient cause for the induction of premature labor. $I$ do not regard albumin as a pathognomonic symptom of approaching eclampsia. But we all feel safer if the urine does not show albumin in these cases. Its presence is suspicious. I should want other evidence than the presence of albumin in order to bring on premature labor. In the paper I have ?resented to you I have merely given my own experience, and I must say that $I$ have better results if $I$ bring on premature labor, particularly when the prodromic indications are sufficiently severe to warrant it.

\section{FIBROID TUMORS OF THE UTERUS, WHEN AND HOW TO OPERATE.}

Read in the Section on Obstetries and Diseases of Women at the Fortyseventh Anual il eeting of the Ameri'rn Medical Association,
at Atlanta, Ga., May 5-8, 189ti.

BY ALBERT H. TUTTLE, M.D., S.B. CAMBRIDGE, MASS.

It is but a short time ago an opinion was more or less universally maintained that fibroid tumors of the uterus were benign growths. The fact that hysterectomy had such a high mortality at that period made the "cure worse than the disease," and probably did much to place these tumors in this relatively false position.

With improvements in technique and correspond. ingly excellent results in the removal of the uterus, it is no longer necessary or wise to wait until life is in immediate danger before recourse to the radical treatment of fibroids by surgical measures, but before the health of the patient is reduced by hemorrhage, inflammation, chronic suppuration and pain, to a delicate condition, and the dangers of the operation are further increased, as well as the difficulties by the complications of inflammation-pus and adhesionsto say nothing of the degenerative changes, the increase in the size of the tumor, and the effects of pressure on the ureters, there must be a time when certain indications exist for the truly conservative removal of these growths.

That this period for active surgical interference varies greatly in the opinion of various operators from the radical view of Jacobs, who considers that the presence of a fibroid tumor is itself sufficient reason for the removal of the uterus, to the conservative extremes of the electro-therapeutists, needs only to be mentioned, and it is this fact that has prompted me to express the conclusions derived from my personal experience in the treatment of these growths.

Small sub-serous tumors, when they give rise to pain and tenderness, interfere with motion and locomotion, produce reflex and nervous disturbances, or directly by pressure make more or less troublesome the functions of menstruation, defecation and micturition, should be removed. They are best extracted through the abdominal route, by enucleation. The peritoneal flaps are sewed together with fine animal sutures and the uterus dropped into place.

Whenever an interstitial fibroid has reached any considerable development, or a number of small ones have increased the size of the uterus above that of the fist, the uterus and fibroids should be removed en masse, to prevent changes which sooner or later will surely result from the constant pressure exerted upon 
the uterus. Baer's supra-vaginal amputation of the uterus is the simplest operation.

Whenever the fibroid condition is complicated by inflammatory changes, either in the growth itself, the pelvic cellular tissue, uterus, tubes or ovaries, a total extirpation should be performed. I know of no better method for the complete removal of the uterus than the vagino-abdominal method, which I have devised and already described. Unless the symptoms are urgent I would recommend a long period of rest and local treatment as a preliminary to surgical treatment (six weeks to two months), as by this means much of the infiltration and subsequent danger of auto-infection will be avoided.

Whenever the common complications of a fibroid tumor, pain, hemorrhage and serous discharges are uncontrolable, the uterus should be removed. Unless there are extra-uterine complications, Baer's supravaginal amputation should be preferred.

When a fibroid uterus is complicated with pregnancy, and one or more nodules rapidly increase in size, early interference is demanded. Often the fibroids can be removed without disturbing the contents of the uterus; but an early removal may necessitate killing of the fetus, in which case operators will become divided in their course of procedure; some, to give the mother the best chance for life, will remove uterus and fibroids early, or the contents of the uterus, others will wait until near term and do a Cæsarean or Porro's operation, while a few will do nothing until labor sets in, when they will attempt to raise the tumor out of the pelvis so as to admit the passage of the child. Each case demands special consideration; a multi-nodular fibroid uterus is less likely to be disabled to the extent of complete incapacity for performing the function of expulsion of a fetus, and at most make the condition of delivery simply one of inertia uteri; a single fibroid is apt to increase to dimensions sufficiently great for the mechanical obstruction of delivery, and at term will nearly always be found in the pelvis or the lower segment of the uterus, a point it has reached from the force of gravity, no matter where the place of origin.

One or more growths of hen's egg size may not seriously interfere with full term delivery, but a growth of this dimension in the early months of pregnancy is more than likely to attain dangerous proportions before the time has arrived for the natural expulsion of the child. If the growths alone are removed a subsequent ventral hernia will usually more or less rapidly follow the final delivery at the site of the abdominal incision, and must be considered among the indications for the removal of the fetus.

The danger of sepsis from auto-infection is increased in the abortion of a fibroid uterus.

When a fibroid uterus is complicated with pregnancy, the problem to be solved is not one of comfort or health, but the serious condition of life or death, therefore surgical interference should not take place until a study of the condition of the patient and the relations of the tumor and uterus show clearly to the operator that there are greater chances of life with operation than without.

When failure in health occurs in a woman having a fibroid uterus, and no cause for the same can be found, unless it be the condition of the uterus, for which the tonic and medicinal methods of treatment are unsuccessful, hysterectomy, a supra-vaginal amputation, should be performed as dernier ressort.
CONSTIPATION: SOME OF ITS EFFECTS AND ITS NON-MEDICINAL

\section{TREATMENT.}

Read in the Section on Practice of Medicine, at the Forty seventh Annual Meeting of the American Medical Association. at tlanta, Gil., May 5-8.1896.

BY E. S. PETTYJOHN, M.D. ALMA, $\mathrm{M}: \mathrm{CH}$.

The large number of people suffering from constipation and its effects, and the clinical difficulty met in obtaining relief therefrom, leads to the conclusion that the full signification of this affliction and its deleterious influence are not comprehended either by the people or the profession.

In its reciprocal influence this condition sustains both a causative and a resultant relation. Constipation as a functional disorder may be defined as an abnormal condition of the great colon and the entire intestinal tract, manifested by the unusual retention of excrementitious material, both as to quantity and time of evacuation. It may be owing either to diminished action of the muscular coat, to the diminished secretion from the mucous membrane, or both, to defective innervation, to habits and occupation, climate or diet, in addition to which there seems to be an infinite variety of causes.

A study of the history of 300 cases shows that about 60 per cent. of patients are suffering from this ailment, and that the number is proportionately larger among women than among men.

Congenital constipation may occur dependent upon the anatomy of the colon, the water being absorbed by a reduplication of the colon itself, especially the descending part, as that part is longer in infants in proportion as compared with the ascending and transverse, and hence liable, by being crowded down into the pelvis, to flexures and reduplications that favor impaction.

In adults the transverse colon is most liable to variation in length and position, and being smaller in diameter than the ascending, aside from congenital malformation, may be bent downward almost to the pubes by long continued distension, forming a sharp bend at the sustentaculum hepatis and at the splenic flexure, as has been found in two cases under the writer's observation.

In considering this condition, we regard individual habit and idiosyncrasy and compare with the rule, as persons do become constipated without notable or sensible inconvenience to themselves, without manifesting any of the local symptoms, and while having regular and apparently sufficient evacuations.

We come to the consideration of constipation more on account of its relation to other ailments and the entire system, than because of the mere inconvenience induced. The intestines and colon and the rectum are considered as digestive, eliminating and secreting organs, and also as organs of absorption.

With their great extent of retiform tissue enclosing a multitude of blood vessels and nerve ramifications, with their mucus follicles and valves of retardation, with their glands, lacteals, arteries and veins, the powers of retention and absorption belonging to this tripod are beyond compare. The wonder is that all the contents which enter are not taken into the circulation.

While the colon and rectum have not the digestive office we have long supposed, their absorptive power is greater than has been thought, and the quantity 